\title{
Clinical characteristics of cognitive deficits in major depressive disorder: a 6-month prospective study
}

\author{
YAJUAN Ji1,2 \\ https://orcid.org/0000-0001-8722-2682 \\ WeIHUI Li² \\ https://orcid.org/0000-0001-5257-6122 \\ BANGSHAN LiU2 2 \\ https://orcid.org/0000-0002-9355-2183 \\ JiN LiU2 \\ https://orcid.org/0000-0001-7959-1541 \\ YUMENG Ju2 \\ https://orcid.org/0000-0002-9745-9840 \\ Mi WANG 2 \\ https://orcid.org/0000-0002-4205-9831 \\ YANChaO $\mathrm{CHEN}^{2}$ \\ https://orcid.org/0000-0003-2222-5633 \\ LINGJIANG $\mathrm{LI}^{2}$ \\ https://orcid.org/0000-0002-8775-7852 \\ 'Department of Psychiatry, Xiamen Xianyue Hospital, Xiamen, Fujian, China. \\ 2 Mental Health Institute of Central South University, China National Clinical Research Center on Mental Disorders (Xiangya), China National Technology Institute on Mental Disorders, \\ Hunan Technology Institute of Psychiatry, Hunan Key Laboratory of Psychiatry and Mental Health, Changsha, Hunan, China.
}

Received: 02/01/2019 - Accepted: 06/04/2020

DOl: 10.1590/0101-60830000000241

\begin{abstract}
Background: Previous studies have shown that major depressive disorder (MDD) is associated with a variety of cognitive deficits, which can persist even in remitted states. Nevertheless, the relationship between the cognitive and affective symptoms in depression remains obscure. The aim of the present study was to explore the clinical characteristics and correlates of the cognitive deficits in patients with MDD. Methods: Clinical and neuropsychological assessments were conducted at baseline and 6-month follow-ups. The severity of the disease and the effect of treatment were assessed with the Hamilton Depression Scale-17. Neuropsychological tests, including the digital symbol substitution test and digit span test, were administered to 67 depressed patients and 56 healthy participants. Results: MDD patients showed impairments in memory, attention, and executive function at baseline. After the 6-month treatment phase, patients in remission showed significant alleviation of these cognitive deficits, although impairments in attention and executive function were still present when compared to controls. Discussion: Significant cognitive deficits are present in MDD. The speed of remission of cognitive functions seems to be slower than and inconsistent with emotional symptoms, which provides new support for the argument that cognitive deficits are independent factors from the emotional symptoms in MDD.
\end{abstract}

Ji Y et al. / Arch Clin Psychiatry. 2020;47(4):101-5

Keywords: Major depressive disorder, cognitive deficits, executive function, remission.

\section{Introduction}

Major depressive disorder (MDD) is a common psychiatric disease with a high frequency of recurrence, affecting millions of people worldwide. In addition to the well- defined depressive symptoms, such as low mood, patients suffering from MDD consistently complain about cognitive disturbances, which significantly exacerbate the burden of this illness ${ }^{1-3}$. Among the cognitive symptoms, impairments in attention, working memory, processing speed, and executive function are often reported.

A considerable number of studies have shown that cognitive deficits are promising candidates for the core symptoms of MDD, and independent risk factors for poor prognosis ${ }^{4-6}$. Specifically, some studies have found that the progression of cognitive symptoms is inconsistent with that of depressive symptoms, that the recovery of cognitive deficits is slower than the remission of emotional symptoms, and finally, cognitive symptoms have been repeatedly reported in participants with subclinical depression as well as participants at high risk of developing depression ${ }^{7-9}$. All of these findings suggest that the cognitive symptoms are independent of the emotional symptoms in MDD. Therefore, some researchers have proposed that cognitive deficits are trait rather than state characteristics, namely endophenotypes, in $\mathrm{MDD}^{10,11}$. Wieland-Fiedler et al. observed deficits in executive function and sustained attention when residual symptoms were controlled for statistically, suggesting that they may represent trait markers for MDD 12

Nevertheless, existing studies have generally focused on pretreatment cognitive function assessments and have mostly been comparative studies with healthy controls. To date, there are few prospective studies comparing cognitive function before and after treatment. Furthermore, most studies have not restricted the types of medication taken by participants, thus making it difficult to control for the effect of multi-drug combinations on cognitive function. Given the above considerations, our study aimed to explore the relationship between cognitive function and depressive symptoms in MDD by assessing participant performance on neuropsychological 
tests before and after treatment with a single antidepressant. In addition, we analyzed the relationship between cognitive deficits and gender, level of education, severity of depression, age of onset, episodes, and illness duration to explore the potential correlate affecting cognitive function in MDD.

\section{Methods}

\section{Participants}

\section{MDD group}

A total of 67 drug-naive MDD patients were recruited from the outpatient clinic at the Second Xiangya Hospital of Central South University. Participants had to meet the following inclusion criteria: (1) outpatients; (2) aged 18-65 years old; (3) meeting the International Statistical Classification of Diseases and Related Health Problems 10 criteria for a single or recurrent major depressive episode with no history of mania or hypomania (the diagnosis was established by a consultant psychiatrist after assessment with the Mini International Neuropsychiatric Interview); (4) starting a new antidepressant monotherapy (either the first one or an altered one) with a washout period of at least four weeks; (5) willing to provide informed consent. Patients were excluded if they had any of the following conditions: (1) any other primary psychiatric disorder; (2) hyperthyroidism or hypothyroidism; (3) receiving electroconvulsive therapy or repeated transcranial magnetic stimulation; (4) women who were pregnant, less than 6 months post-childbirth, or lactating; (5) experiencing severe suicidal tendencies.

\section{Healthy control group}

A total of 56 healthy controls were recruited during the same period. The inclusion criteria were (1) aged 18-65 years old (male or female); (2) having a Hamilton Depression Scale-17 (HAMD-17) score lower than 7. The remaining criteria were the same as in the MDD group.

All research protocols were approved by the Xiangya second hospital of central south university, and all participants gave written informed consent.

\section{Measurements}

\section{Assessment tools}

(1) Clinical assessment

The HAMD-1713 was used to assess the severity of depression. It includes five subfactors: relating to retardation, insomnia, cognitive dysfunction, anxiety/somatization, and weight loss.

\section{(2) Neuropsychological testing}

The digit span test ${ }^{14}$ measures participants' attention and short-term memory. It consists of recalling digit spans forward and backward. The forward test mainly assesses working memory, while the backward test assesses attention switching capacity.

The digital symbol substitution test (DSST) ${ }^{14,15}$ is part of the Wechsler Intelligence Scale (digital symbol coding). It measures cognitive function, including executive function, visual orienting, processing speed, attention, and memory function, which in combination represent the ability to conduct complex cognitive multitasking.

\section{Assessment methods}

The MDD group received one type of selective serotonin reuptake inhibitors (SSRIs) from baseline for a period of 6 months. The HAMD-17 score was used to assess the severity of depression, and neuropsychological tests were employed to measure cognitive function in MDD before and after the treatment (i.e., at the 8- week and 6-month follow-up assessments). The control group also completed the above assessments. The clinical assessments and cognitive tests were completed by two researchers, with a consensus (Kappa) coefficient of 0.90 .

Remission was defined as a period of at least 2 months during which the individual was diagnosed as being in clinical remission with a HAMD-17 score $\leq 7$. After the 6 -month period of treatment, we successfully conducted follow-up assessments with 44 patients. Among these, 17 patients had achieved remission whereas the remaining 27 patients did not meet the criterion for remission.

\section{Statistical analysis}

SPSS 22.0 statistical software was used to conduct chi-square tests, independent sample $t$ tests, paired $t$ tests, covariance analyses, and linear regression analyses. The significance level for all analyses was set at $P<0.05$.

\section{Results}

\section{Characteristics of cognitive function in MDD}

As shown in Table 1, the total sample was composed of 67 MDD patients and 56 healthy controls. Except for the severity of the illness, no statistical differences were found between the MDD patients and healthy controls in terms of demographic and clinical characteristics at baseline. At the 8-week follow-up, data could not be obtained from 19 patients. At the 6-month follow-up, data could not be obtained from 4 patients. Further analyses were conducted on 44 patients, 27 of whom were in clinical remission. As can be seen in Table 2 , at baseline the depressed patients experienced more severe emotional symptoms and demonstrated poorer performance on each neuropsychological test compared to the healthy controls. At the 8-week follow-up assessment, the emotional symptoms and cognitive deficits in the patients showed obvious alleviation relative to baseline, although the DSST and backward digit span performance were both still significantly poorer than in controls. At the 6-month follow-up assessment, the depressed patients still had poorer performance on the DSST and backward digit span than the controls.

As shown in Table 3, the non-remitted patients demonstrated poorer performance on all neuropsychological tests except the forward digit span at the 6-month follow-up as compared to healthy controls. However, the remitted patients had poorer performance only on the DSST, achieving a comparable level of performance to that of the healthy controls on the forward and backward digit span at the 6-month follow-up.

\section{Clinical correlates of the cognitive deficits in MDD}

We used linear regression analysis with cognitive function at baseline as the dependent variable and various clinical characteristics of the patients (gender, age at onset, level of education, number of episodes, illness duration, and severity of depression) as the independent variables.

As shown in Table 4, DSST performance was positively correlated with level of education and negatively correlated with the age of depression onset and illness duration. The forward digit span was positively correlated with level of education and negatively correlated with the age of depression onset and number of episodes. The backward digit span was positively correlated with level of education and negatively correlated with HAMD-17 scores. Linear regression analysis showed that level of education, age of depression onset, and illness duration explained $37.0 \%$ of the variance in DSST performance; level of education, age of depression onset, and number of episodes explained $30.6 \%$ of the variance in forward digit span performance; and level of education and HAMD-17 scores explained $14.2 \%$ of the variance in backward digit span performance. 
Table 1. Demographic and clinical characteristics for all participants (mean \pm SD)

\begin{tabular}{|l|c|c|c|c|}
\hline & $\operatorname{MDD}(\mathrm{N}=67)$ & Controls (N=56) & Statistics (t/ $/$ 2) & $P$ \\
\hline Age (years) & $31.00 \pm 9.84$ & $33.83 \pm 12.83$ & 1.385 & 0.169 \\
\hline Sex (male/female) & $30 / 37$ & $25 / 31$ & 0.374 & 0.241 \\
\hline Education (years) & $13.18 \pm 3.08$ & $13.30 \pm 4.70$ & - & -89 \\
\hline Duration (months) & $16.96 \pm 15.21$ & - & - & - \\
\hline Age of onset (years) & $22.36 \pm 5.39$ & - & - & - \\
\hline Number of episodes & $3.72 \pm 2.17$ & - & - & - \\
\hline First-episode/total & $27 / 67$ & - & 45.934 & \\
\hline HAMD-17 & $20.91 \pm 2.51$ & $2.46 \pm 1.94$ & & -000 \\
\hline
\end{tabular}

Table 2. Cognitive characteristics of the depressed patients at baseline and after treatment

\begin{tabular}{|c|c|c|c|c|c|c|}
\hline & \multirow{2}{*}{$\begin{array}{l}\text { Baseline } \\
(\mathrm{N}=67)\end{array}$} & \multirow{2}{*}{$\begin{array}{l}\text { Follow-up (8-week) } \\
\qquad(\mathrm{N}=48)\end{array}$} & \multirow{2}{*}{$\begin{array}{l}\text { Follow-up (6- month) } \\
\qquad(\mathrm{N}=44)\end{array}$} & \multirow{2}{*}{$\begin{array}{l}\text { Controls } \\
(\mathrm{N}=56)\end{array}$} & \multicolumn{2}{|c|}{ Statistics } \\
\hline & & & & & $\mathrm{F}$ & $P$ \\
\hline HAMD-17 & $20.91 \pm 2.51 *$ & $9.73 \pm 2.91^{*} \#$ & $7.57 \pm 3.37^{*} *$ & $2.46 \pm 1.94$ & 523.578 & $<0.001$ \\
\hline \multicolumn{7}{|c|}{ Digital symbol substitution test (DSST) } \\
\hline Correct & $49.79 \pm 10.93^{*}$ & $58.02 \pm 9.80 * *$ & $60.64 \pm 11.05^{*} *$ & $65.82 \pm 13.60$ & 20.852 & $<0.001$ \\
\hline \multicolumn{7}{|c|}{ Digit Span Test } \\
\hline Forward & $8.64 \pm 1.08^{\#}$ & $9.31 \pm 0.75^{*}$ & $9.59 \pm 0.92^{*}$ & $9.64 \pm 1.17$ & 12.656 & $<0.001$ \\
\hline Backward & $4.85 \pm 0.97 *$ & $5.48 \pm 1.01 * *$ & $5.48 \pm 0.90^{*} \#$ & $6.36 \pm 1.54$ & 17.665 & $<0.001$ \\
\hline
\end{tabular}

Note: ${ }^{*} P<0.05$ vs. baseline. ${ }^{*} P<0.05$ vs. controls.

Table 3. Cognitive characteristics of the remitted and non-remitted depressed patients at 6-month follow-up

\begin{tabular}{|c|c|c|c|c|c|}
\hline & \multirow{2}{*}{$\begin{array}{l}\text { Non-remitted } \\
(\mathrm{N}=17)\end{array}$} & \multirow{2}{*}{$\begin{array}{l}\text { Remitted } \\
(\mathrm{N}=27)\end{array}$} & \multirow{2}{*}{$\begin{array}{l}\text { Controls } \\
(\mathrm{N}=56)\end{array}$} & \multicolumn{2}{|c|}{ Statistics } \\
\hline & & & & $\mathrm{F}$ & $P$ \\
\hline \multicolumn{6}{|c|}{ Digital symbol substitution test (DSST) } \\
\hline Correct & $54.06 \pm 11.65^{*}$ & $64.85 \pm 8.37$ & $65.82 \pm 13.60$ & 6.392 & 0.002 \\
\hline \multicolumn{6}{|c|}{ Digit span test } \\
\hline Forward & $9.29 \pm 0.85$ & $9.74 \pm 0.94$ & $9.64 \pm 1.17$ & 0.982 & 0.193 \\
\hline Backward & $5.06 \pm 0.90^{*}$ & $5.81 \pm 0.83^{*}$ & $6.36 \pm 1.54$ & 6.926 & 0.002 \\
\hline
\end{tabular}

Note: ${ }^{*} P<0.05$ vs. controls.

Table 4. Linear regression analyses of clinical predictors and cognitive deficits at baseline

\begin{tabular}{|l|l|c|c|c|c|c|c|}
\hline Independent variables & Dependent variables & B & $\beta$ & Constant & $R^{2}$ & $\mathrm{~F}$ & $\mathrm{P}$ \\
\hline DSST & Age of onset & -0.465 & -0.407 & 82.233 & 0.370 & 9.192 & 0.001 \\
& Duration & -0.176 & -0.249 & & & 0.040 \\
& Education & -7.842 & -0.377 & & & 0.002 \\
\hline Forward digit span & Age of onset & -0.141 & -0.300 & 7.670 & 0.306 & 8.259 & 0.020 \\
& Education & 0.182 & 0.444 & & & $<0.001$ \\
& Frequency & -0.521 & -0.241 & & & 0.040 \\
\hline Backward digit span & Education & 0.082 & 0.260 & 3.964 & 0.142 & 5.298 & 0.030 \\
& HAMD & -0.416 & -0.235 & & & \\
\hline
\end{tabular}

\section{Discussion}

\section{The characteristics of cognitive deficits in MDD}

The results of the present study indicate that significant cognitive deficits are present in MDD, including deficits in executive function, attention, and memory. At 6-month follow-up after treatment, patients demonstrated significant improvement on all three neuropsychological tests, among which the forward digit span recovered to the level of healthy control, while the backward digit span and digit symbol substitution test performance remained suboptimal. These findings suggest that the trajectories of different aspects of cognitive function in MDD are asynchronous, and some may be independent of emotional symptoms. Specifically, the improvement in working memory (as tested by the forward digit span task) was the most obvious, as patients exhibited improvement in this function even when they did not achieve clinical remission. The speed of the improvement in executive function (as tested by the DSST) was slower since only patients with clinical remission showed recovery from impairment on this task. Meanwhile, the recovery of attention switching (as tested by backward digit span) was minimal and had little relation to emotional symptoms, since the deficits in attention persisted even in the patients who had achieved clinical remission.

Our results are consistent with a considerable number of previous literature reviews

and longitudinal studies. For instance, Lee and colleagues found that attention and executive functioning deficits were not associated with depression severity, suggesting that they may function as important trait markers for MDD ${ }^{16}$. This is consistent with a recent 
study by Douglas et al. demonstrating that psychomotor speed improved in successfully-treated in-patients with severe depression whereas attention and executive dysfunction persisted despite remission of depressive symptoms ${ }^{17}$.

However, the findings of some studies are inconsistent with the present results. For instance, a recent study found that executive dysfunction appeared to be a state-specific marker of MDD that is related to depression severity and is not present in remission ${ }^{18,19}$. Maalouf et al. found that the executive dysfunction of adolescent patients was correlated with the severity of depression and gradually returned to normal level in remission ${ }^{20}$. One possibility is that the adolescent brain may be better able to recover from executive function deficits associated with depression. These inconsistencies in findings could be partly due to the testing of cognitive dysfunction in MDD being limited by heterogeneous demographic features (such as age and educational attainment), clinical characteristics (such as first, multi-episode, or chronic depression, severity of symptoms, age of disease onset, substance use, and comorbid medical conditions), and treatment assignments. In our study, some patients were given benzodiazepines, while Buffett's study found that benzodiazepines produce profound increases in subjective sedation, slow psychomotor and cognitive processing speed, and lead to attentional impairments ${ }^{21}$. Together, these variables likely play a major role in contributing to the inconclusive findings reported in the existing studies. In addition, the lack of standard tools for assessing cognition in MDD and the variety of neuropsychological tests employed in different investigations hinder clear comparisons between studies.

\section{The correlates of cognitive deficits in MDD}

We found that education level and age of depression onset are major factors associated with cognitive deficits in MDD. Similarly, there are different degrees of correlation between cognitive deficits and episodes, illness duration, and severity of symptoms.

As shown in the present study, working memory, attention switching, and executive function are consistently associated with level of education, which indicates that more highly educated patients show enhanced cognitive reserve capacity Many previous studies are consistent with our results. For instance, Jarema et al. showed that more highly educated patients demonstrated better performance on all the assessed measures of cognitive function ${ }^{22}$. In addition, a study focusing on cognitive functioning in healthy older adults found that participants with a medium or high level of education performed better on cognitive tests than participants with a low level of education. In other words, people with a high level of education may have a greater cognitive reserve capacity than people with a lower level of education ${ }^{23}$.

Since the age of depression onset is closely correlated with different aspects of cognitive function, late-onset depression may be associated with more severe cognitive impairments ${ }^{24,25}$. A metaanalysis found that patients with late-onset depression showed greater reductions in processing speed and executive function than patients with early-onset depression and controls16,26. One explanation for this may be that cognitive function, especially executive function, may gradually decrease with age. In addition, late-onset depression has been associated with cerebrovascular disease risk factors, which may also play an important role in the development of cognitive impairment 27 .

However, several limitations must be acknowledged. In this study, patients were treated with a single antidepressant, meanwhile some also received benzodiazepine in combination, but we did not specifically discuss the type and dose of antidepressant used, which may impact negatively on cognitive function. We did not discuss comorbidities, marital status and family history of mental illness. The above factors may affect cognitive function and social function to some extent. The neuropsychological assessment tools in this study are relatively limited, involving only executive function, attention and memory, which cannot comprehensively evaluate the cognitive function of patients with depression. Therefore, more comprehensive assessment tools should be included in future studies.

In conclusion, patients with MDD may exhibit impairments in various cognitive domains, including attention, executive function, and memory. While, the trajectories of different aspects of cognitive function in MDD are asynchronous during remission, and some may be independent of emotional symptoms. Cognition is thus a key target in the treatment of depression especially with respect to early recognition and intervention. In view of the above findings, it is appropriate to conclude that attention deficits and executive dysfunction may serve as trait markers of MDD, while working memory deficits may be regarded as a state marker of MDD. Whether cognitive dysfunction is a state- or trait-related phenomenon in depression is a subject of ongoing debate. A promising approach to clarifying this matter is the additional consideration of clinical and demographic factors that are known to be related to cognitive performance. This is an important goal since cognitive deficits contribute to long-term functional outcomes, and MDD patients experiencing deficits may show less compliance with treatment and an increased risk for suicide.

\section{References}

1. Bortolato B, Carvalho AF, McIntyre RS. Cognitive dysfunction in major depressive disorder: a state-of-the-art clinical review. CNS Neurol Disord Drug Targets. 2014;13(10):1804-18.

2. Fehnel SE, Forsyth BH, DiBenedetti DB, Danchenko N, François C, Brevig T. Patient-centered assessment of cognitive symptoms of depression. CNS Spectr. 2016;21(1):43-52.

3. Conradi HJ, Ormel J, de Jonge P. Presence of individual (residual) symptoms during depressive episodes and periods of remission: a 3-year prospective study. Psychol Med. 2011;41(6):1165-74.

4. Paelecke-Habermann Y, Pohl J, Leplow B. Attention and executive functions in remitted major depression patients. J Affect Disord. 2005;89(13):125-35.

5. Darcet F, Gardier AM, Gaillard R, David DJ, Guilloux JP. Cognitive Dysfunction in Major Depressive Disorder. A Translational Review in Animal Models of the Disease. Pharmaceuticals (Basel). 2016;9(1):9.

6. Hinkelmann K, Moritz S, Botzenhardt J, Riedesel K, WiedemannK, Kellner M, et al. Cognitive impairment in major depression: association with salivary cortisol. Biol Psychiatry. 2009;66(9):879-85.

7. Svendsen AM, Kessing LV, Munkholm K, Vinberg M, Miskowiak KW Is there an association between subjective and objective measures of cognitive function in patients with affective disorders? Nord J Psychiatry. 2012;66(4):248-53.

8. Roiser JP, Sahakian BJ. Hot and cold cognition in depression. CNS Spectr. 2013;18(3):139-49.

9. Zlatar ZZ, Moore RC, Palmer BW, Thompson WK, Jeste DV. Cognitive complaints correlate with depression rather than concurrent objective cognitive impairment in the successful aging evaluation baseline sample. J Geriatr Psychiatry Neurol. 2014;27(3):181-7.

10. Jaeger J, Berns S, Uzelac S, Davis-Conway S. Neurocognitive deficits and disability in major depressive disorder. Psychiatry Res. 2006;145(1):39-48.

11. Porter RJ, Bourke C, Gallagher P. Neuropsychological impairment in major depression: its nature, origin and clinical significance. Aust N Z J Psychiatry. 2007;41(2):115-28.

12. Weiland-Fiedler P, Erickson K, Waldeck T, Luckenbaugh DA, Pike D Bonne $\mathrm{O}$, et al. Evidence for continuing neuropsychological impairments in depression. J Affect Disord. 2004;82(2):253-8.

13. Hamilton M. A rating scale for depression. J Neurol Neurosurg Psychiatry. 1960;23(1).

14. Wechsler D. Wechsler Adult Intelligence Scale. New York: Springer; 1997

15. Zihl J, Fink T, Pargent F, Ziegler M, Bühner M. Cognitive Reserve in Young and Old Healthy Subjects: Differences and Similarities in a Testing-the-Limits Paradigm with DSST. PLoS One. 2014;9(1):e845901.

16. Herrmann LL, Goodwin GM, Ebmeier KP. The cognitive neuropsychology of depression in the elderly. Psychol Med. 2007;37(12):1693-702.

17. Douglas KM, Porter RJ. Longitudinal assessment of neuropsychological function in major depression. Aust N Z J Psychiatry. 2009;43(12):1105-17.

18. Bortolato B, Miskowiak KW, Köehler CA, Maes M, Fernandes BS, Berk $\mathrm{M}$, et al. Cognitive remission: a novel objective for the treatment of major depression? BMC Med. 2016;14:9. 
19. Talarowska M, Zajaczkowska M, Galecki P. Cognitive functions in first-episode depression and recurrent depressive disorder. Psychiatr Danub. 2015;27(1):38-43.

20. Maalouf FT, Brent D, Clark L, Tavitian L, McHugh RM, Sahakian BJ, et al. Neurocognitive impairment in adolescent major depressive disorder: State vs. trait illness markers. J Affect Disord. 2011;133(3):625-32.

21. Buffett-Jerrott S, Stewart S. Cognitive and Sedative Effects of Benzodiazepine Use. Curr Pharm Des. 2002;8(1):45-58.

22. Jarema M, Dudek D, Czernikiewicz A. [Cognitive dysfunctions in depression - underestimated symptom or new dimension?]. Psychiatr Pol. 2014,48(6):1105-16.

23. van Hooren SA, Valentijn AM, Bosma H, Ponds RW, van Boxtel MP, Jolles J. Cognitive functioning in healthy older adults aged 64-81: a cohort study into the effects of age, sex, and education. Neuropsychol Dev Cogn B Aging Neuropsychol Cogn. 2007;14(1):40-54.
24. Hasselbalch BJ, Knorr U, Kessing LV. Cognitive impairment in the remitted state of unipolar depressive disorder: a systematic review. J Affect Disord. 2011;134(1-3):20-31.

25. Bora E, Harrison BJ, Davey CG, Yücel M, Pantelis C. Meta-analysis of volumetric abnormalities in cortico-striatal-pallidal-thalamic circuits in major depressive disorder. Psychol Med. 2012;42(4):671-81.

26. Bora E, Harrison BJ, Yücel M, Pantelis C. Cognitive impairment in euthymic major depressive disorder: a meta-analysis. Psychol Med. 2013;43(10):2017-26.

27. Sheline YI, Barch DM, Garcia K, Gersing K, Pieper C, Welsh-Bohmer $\mathrm{K}$, et al. Cognitive function in late life depression: relationships to depression severity, cerebrovascular risk factors and processing speed. Biol Psychiatry. 2006;60(1):58-65. 